Revue d'histoire de l'Amérique française

REVUE D'HISTOIRE DE L'AMÉRIQUE FRANÇAISE

\title{
Monsieur Charles de Bellefeuille, missionnaire de l'Outawais (1836-38)
}

\section{Yvon Charron}

Volume 5, numéro 2, septembre 1951

URI : https://id.erudit.org/iderudit/801698ar

DOI : https://doi.org/10.7202/801698ar

Aller au sommaire du numéro

Éditeur(s)

Institut d'histoire de l'Amérique française

ISSN

0035-2357 (imprimé)

1492-1383 (numérique)

Découvrir la revue

Citer cet article

Charron, Y. (1951). Monsieur Charles de Bellefeuille, missionnaire de l'Outawais (1836-38). Revue d'histoire de l'Amérique française, 5(2), 193-226. https://doi.org/10.7202/801698ar d'utilisation que vous pouvez consulter en ligne.

https://apropos.erudit.org/fr/usagers/politique-dutilisation/ 


\section{MONSIEUR CHARLES DE BELLEFEUILLE MISSIONNAIRE DE L'OUTAWAIS}

$$
(1836-38)
$$

Le diocèse de Montréal venait de naître lorsque Monsieur Ignace Bourget, pour lors secrétaire de Monseigneur Lartigue, écrivait les lignes qui suivent au curé de la Petite Nation, Monsieur Pascal Brunet: "La mission de Témiskaming est enfin résolue par la décision du Docteur Stevenson qui déclare M. [Charles de] Bellefeuille assez fort pour aller dans cette terre infidèle"'. L'initiative émanait, en fin de compte, du nouvel et premier évêque de Montréal qui avec son esprit apostolique, nourrissait, depuis son sacre survenu le 21 janvier 1821, le projet de "faire adorer J[ ésus ] $\mathrm{C}$ [ hrist ] par les tribus infidèles qui habitent la partie supérieure du district de Montréal, vers le nord-ouest"'2. Ce désir, il reparaît périodiquement dans la correspondance de Monseigneur Lartigue, qui, au surplus, s'en exprime toujours comme d'une chose qui lui tient au cœur, soit qu'il se réclame du témoignage de Monseigneur Provencher pour presser l'envoi de missionnaires ${ }^{3}$, soit qu'il s'impatiente et s'indigne des lenteurs mises par les Sulpiciens à prendre la route ${ }^{4}$.

1. Cette lettre fut en effet écrite de Varennes, le 29 mai 1836. Or l'on sait que la bulle de Grégoire XVI érigeant le district de Montréal en diocèse autonome porte la date du 13 mai de la même année, Rapport de l'archiviste de la Province de Québec, [ dorénavant cité RAPQ] (1944-45), 190-91.

2. J.-B. Dupuy, "Journal d'un voyage fait à Témiskaming en 1836" [ dorénavant cité Journal d'un voyage ], dans Rapport de l'Association de la Propagation de la Foi établie à Montréal en vertu d'un indult du Saint-Siège et annoncée au diocèse par le mandement du 18 avril 1836 [ dorénavant cité RAPF] 1 (1839): 25. 317.

3. "Mgr Lartigue à M. [ Pascal ] Brunet", le 31 octobre 1835, RAPQ (1943-44),

4. Mgr Lartigue avait demandé au Supérieur du Séminaire d'envoyer M. Flavien Durocher, alors chargé de la mission algonquine du Lac (Oka). M. Quiblier avait refusé pour des raisons que nous ignorons. C'est alors que Mgr Lartigue fait part de son échec à Mgr Panet et ajoute qu'en agissant ainsi M. Quiblier va “contre les titres mêmes des Sulpiciens en ce pays, leurs biens leur ayant été donnés principalement pour la conversion des sauvages". "Mgr Lartigue à Mgr Panet, 12 mai 1832, Correspondance de Monseigneur Lartigue", RAPQ (1942-43), 145. Apparemment les Sulpiciens du Canada ont eu quelque peine à trouver leur vocation au pays puisque huit ans plus tard, en 1840, Mgr Bourget, devenu évêque de Montréal, remercie le 
Sous des dehors assez humbles, l'entreprise était d'importance. Car, à toutes fins pratiques, c'était chez des peuplades infidèles que Monsieur de Bellefeuille allait "missionner". Il est bien vrai que, tôt sous le régime français, les lacs Témiskaming et Abitibi avaient connu le passage du missionnaire; également admis le fait qu'en 1686, le Père Antoine Silvy accompagnait Iberville dans l'expédition menée par celui-ci contre les établissements anglais de la baie James ${ }^{5}$; enfin il parait sûr aussi que, même après le traité d'Utrecht, au cours de ces cinquante années qui s'écoulèrent de 1713 à 1763, le nord de l'Outawais a été, au moins occasionnellement, visité par le prêtre catholique ${ }^{6}$.

Mais, quoi qu'il en soit, lorsque en juin 1836, Monsieur Charles Lefèvre de Bellefeuille quitte Montréal avec la mission de prêcher la foi aux indiens du lac Témiskamingue, ce sont bien des infidèles, des p̀aiens authentiques qu'il s'agit d'évangéliser:

Vous m'avez si spécialement recommandé de vous faire une relation détaillée de notre voyage à Témiskaming, ecrit à Mgr Lartigue Jean-Baptiste Dupuy, le compagnon de Bellefeuille, - et de vous rendre compte des besoins spirituels

même Monsieur Quiblier de ce que celui-ci a accepté la fondation du Grand Séminaire diocésain et ajoute la remarque suivante: "Depuis 200 ans que votre Séminaire est établi dans ce pays, il a fait sans doute et il fait encore beaucoup de bonnes œuvres, mais il ne faisait pas la sienne. Le voici maintenant en possession de son bien et de son héritage." "Mgr Bourget à M. Quiblier, ler novembre 1840", Archives du Grand Séminaire de Montréal.

5. C. de Rochemonteix, Les Jésuites et la Nouvelle-France au XVIIe siècle, (3 vols, Paris, 1895-1896), 3: 269. "Après avoir remonté l'Ottawa jusqu'au lac Témiscamingue et traversé celui-ci dans sa longueur, - commente Monsieur Guy Frégault, - le détachement prendra les rivières Blanche, Windigo et Kanasula et tombera dans la rivière Abitibi, dont il suivra le cours jusqu'au lac du même nom, où le commandant fera construire un poste dans les premiers jours de juin.," Iberville le Conquerant (Montréal, 1944), 91.

6. Monsieur Frégault fait observer, ibid., 91, que "les traiteurs canadiens fr6́quentèrent et occupèrent re poste - [ celui d'Abitibi ] - jusqu'en 1763, ainsi que ceux de Mistassini et de Nemiscau. Le traité d'Utrecht, continue-t-il, ne céda aux Anglais que les postes situés sur le littoral de la baie James. "Dans la correspondance des gouverneurs et des intendants, il est souvent question de ce poste d'Abitibi. Ceux-ci poussaient les coureurs de bois de ce côté et les exhortaient à prendre tous les moyens possibles pour attirer les sauvages et les empêcher de descendre à la baie James, où ils rencontraient les traiteurs anglais..." Caron, Journal de l'expédition du chevalier de Troyes a la baie d'Hudson en 1686, 54, n. 93. S'il est vrai que pendant cinquante ans les traiteurs et les coureurs de bois furent ainsi orientés vers le Témiskaming et l'Abitibi, il nous parait plus que vraisemblable que les missionnaires y aient aussi paru au moins occasionnellement. 
de celles de vos brebis, que vous nous avez chargés de visiter, de l'état d'abandon où sont les domestiques de la foi dans ces lieux que nous venons de parcourir, des disporitions des infidèles à se faire chrétiens, des obstacles qui s'opposent à leur conversion, des moyens qu'il faudrait prendre pour les amener à la connaissance de J. C., que je ne puis écouter la pensée qui me détourne d'entreprendre une besogne, si fort au dessus de mes forces?

Le dévouement du missionnaire, du reste, révèlerait deux autres choses encore: tout d'abord que par l'un de ses membres, SaintSulpice du Canada allait écrire une autre page de son déjà bel apostolat auprès des indiens d'Amérique septentrionale; et puis, que le tout jeune diocèse de Montréal commençait de donner un effort de propagande missionnaire, qui, tout le long du dix-neuvième siècle, saurait se faire sentir jusqu'en des points du pays aussi distants les uns des autres que les townships de l'Est ${ }^{8}$, les lacs Témiskaming et Abitibi $^{9}$, l'Orégon ${ }^{10}$, enfin les rives du Grand Lac Victoria ${ }^{11}$. Des faits de ce calibre valent que l'on essaie de les revivre.

\section{MISSION DU TÉMISKAMING (20 juin -16 août 1836$)$}

C'était un homme de quarante ans révolus que Monsieur LouisCharles Lefèvre de Bellefeuille [selon toute probabilité il faut lire

7. "J.-B. Dupuy à Mgr Lartigue, au retour de la mission de $\mathrm{M}$. de Bellefeuille en 1836, RAPF, 1 (1839): 24-25.

8. Par exemple: “Missions des townships de l'Est. Lettre de M. La France à M. Hudon", RAPF, 4 (1843): 63-64; aussi: Mission de Durham. Lettre de M. Lafrance à M. Hudon, v.g., RAPF, 4 (1843): 65-68.

9. Par exemple: “Journal d'un voyage fait à Témiskaming en 1836", RAPF, 1 (1839): - [ dorénavant cité Journal d'un Voyage ] - 25-53. Aussi: "Relation d'une mission faite en l'été de 1837, le long de la Rivière de l'Outawa jusqu'au Lac Témiskaming, et au dela jusqu'au Lac d'Abbitibbi dans la district de Monseigneur de Juliopolis" [ dorénavant cité: Relation d'une mission ], RAPF, 2 (1840): 17-72.

10. Consulter sur ce sujet: L. Le Jeune, Dictionnaire général de biographie, histoire, litterature, agriculture, commerce, industrie et des arts, sciences, mœurs, coutumes, institutions politiques et religieuses du Canada (2 vols, Ottawa, 1981), s.v. Walla-Walla, Blanchet (Augustin Magloire).

11. Par exemple: Précis de la relation de la troisieme Mission de Mr Bellefeuille d Temiskaming, Abbitibbi et Grand Lac. [dorénavant cité: Précis de la relation], 2 (1840): 73-88. 
Lefébure $]^{12}$ lorsque le 20 juin 1836, il quittait la mission du Lac (Oka) et prenait le "steamboat" en compagnie de l'abbé JeanBaptiste Dupuy. Il était de santé débile depuis plusieurs années déjà ${ }^{13}$, et cependant Monseigneur Lartigue ne laissait pas de mettre en lui les plus grands espoirs touchant l'évangélisation des peuplades indiennes de l'Outawais-nord.

C'est qu'en effet, quelques années durant (1824-34), il avait travaillé chez les indiens de la réserve d'Oka ${ }^{14}$, et qu'il y avait acquis

12. Notre missionnaire appartenait à la troisième génération de la famille Le Fébure de Bellefeuille dont le premier ancêtre au pays fut Jean François: "Bellefeuille, Jean-François Lefêre de - Originaire de Rennes en Bretagne, il passa en Acadie vers 1700. Marié à une demoiselle Beaudry, M. Lefèvre de Bellefeuille décéda probablement en Acadie." Cf. "Références biographiques canadiennes", Bulletin des Recherches historiques, 52 (1946): 284-86. Nous avons en main l'acte de baptême de M. de Bellefeuille, lequel se lit comme suit: "le douze janvier mil sept cent quatre vingt-quinze par nous curé, soussigné, a été baptisé Louis Charles, né de ce jour du légitime mariage de M. Antoine Lefèvre Bellefeuille et de dame Louise Angélique Dumont. Le parrain a été M. Louis Hugues Hertel de Chambly et la mareine Delle Marie Charles Dumont qui ont signé avec nous ainsi que le père. Hertel Chambly. Charlotte Dumont. A.L. Bellefeuille. B.N. Maillou, prêtre-curé." Archives paroissiales de Saint-Eustache, Qué. Il convient d'ajouter que la famille Lefèvre de Bellefeuille fut illustre par plusieurs de ses membres qui contractèrent de hautes alliances ou bien encore furent des militaires distingués ou des écrivains de renom. Tel, par exemple, celui dont le Bulletin des Recherches historiques donne le signalement suivant: "Joseph-Edouard Lefère de Bellefeuille - Sa Sainteté Pie IX le créa chevalier du corps des zouaves pontificaux canadiens. Il était l'auteur d'un Code civil annote, d'une Thèse sur les mariages clandestins et de l'ouvrage Le Canada et les Zouaves pontificaux, et il avait contribué à fonder le journal l'Ordre et la Revue Canadienne. Ce compatriote distingué est décédé à Montréal le 12 janvier 1926. Dernier survivant des fils de Joseph Lefèvreode Bellefeuille, co-seigneur des Mille-Isles et Cournoyer, et de Caroline Leprohon, le défunt descendait de François Lefèvre de Bellefeuille, seigneur de Pabos et de l'Ance-au-Canard, subdélégué de l'intendant, commandant de la Gaspésie, et qui épousa Marie-Joseph Hertel de Cournoyer, aux Trois-Rivières, en 1749." BRH, 52 (1946): 286; 32 (1926): 170. Consulter aussi: A.-C.-M. de Léry MacDonald, "La famille Le Fébure de Bellefeuille", Revue Canadienne, 20 (1884): 168.

13. En l'année 1930, M. de Bellefeuille qui n'a pourtant encore que 35 ans signe l'affirmation suivante: "Je vous quitte pour écrire à Monsieur Humbert. Ce sera la quatrième lettre après diner. C'est bien assez pour un pauvre valétudinaire comme moi.", Chs de Bellefeuille à M. Comte, 29 mars 1830, A rchives du Séminaire de SaintSulpice de Montrél, v. 2, a. 7, t. 60, carton 9. La lettre de Mgr Bourget à M. Brunet, en date du 29 mai 1836, donne un témoignage équivalent. Cf. note 1 . Il est d'ailleurs significatif d'observer que plusieurs des frères et sœurs du sulpicien sont morts prématurément: Eustache-Antoine, l'aîné, à 43 ans; le missionnaire lui-même, à 43 ans; François-Louis, à 39 ans; Edouard-Louis, à 18 ans; Angélique-Marguerite, à 24 ans; enfin Henri-Nicolas, à 25 ans.

14. Dans ses saillants, la vie de Charles de Bellefeuille se ramène aux dates suivantes: naissance et baptême a Saint-Eustache, le 12 janvier 1795; humanités classiques au Collège de Montréal, 1807-15; études théologiques comme régent au Collège de Montréal, 1815-19; ordination sacerdotale, le 5 juin 1819, à :l'hospice des Sœurs Grises, Place Youville: entrée à Saint-Sulpice en 1821; missionnaire au Lac 
non seulement la connaissance de la langue algonquine, mais aussi l'expérience et l'amour de l'âme indienne, toutes choses qui lui seraient de précieux atouts dans le travail d'évangélisation dont il devenait l'initiateur ${ }^{15}$.

Monsieur Jean-Baptiste Dupuy (1804-79), qui complétait l'équipe missionnaire, était de dix ans plus jeune. Chapelain du jeune évêché de Montréal, il exerçait aussi, au moment où il devint le compagnon de Monsieur de Bellefeuille, la fonction de professeur de théologie au Grand Séminaire-miniature que Monseigneur Lartigue avait fondé en son établissement de Saint-Jacques ${ }^{16}$. Vu son ignorance de la langue algonquine, il n'a pas, dans l'expédition du Témiskaming, la même importance que le sulpicien. Il n'en sera pas moins pour celui-ci un auxiliaire précieux. Et puis il rédigera au jour le jour la chronique de l'expédition, ce qui aura pour résultat de mettre à la disposition de la postérité un document de première main et de belle venue ${ }^{17}$.

Les deux mandataires partaient avec des instructions qu'avait tenu à leur détailler minutieusement l'évêque de Montréal. Qu'on en juge par ce qui suit:

Ils suivront autant que possible la ligne de conduite suivante: 1o. ils planteront une croix sur le site de leur future chapelle dont le patron sera, saint Adalbert, évêque de Prague et martyr, apôtre des Prussiens, des Hongrois et des Polonais, puis ils parcourront en surplis et la clochette à la main la bourgade des sauvages pour leur annoncer qu'ils sont les envoyés du seul vrai Dieu; 2o. ils enseigneront à quelques jeunes

(des Deux-Montagnes) 1824-34; à la paroisse Notre-Dame 1834-38, avec missions chez les indiens de l'Outawais, de 1836 à 1838; mort le 25 octobre 1838.

15. "Relation d'une mission faite en l'été de 1839, le long de la rivière de l'Ottawa jusqu'au lac Témiskaming, etc.. par Charles Ed. Poiré," RAPF, 3 (1841): 3.

16. J.-B.-A. Allaire, Dictionnaire biographique du clergé canadien-français, Les anciens (Montréal, 1910), 196-97. - "Mgr Lartigue à M. Jean-Charles Prince", Montréal, 2 octobre 1832, RAPQ (1942-43), 160.

17. "Mgr Lartigue à MM. Charles de Bellefeuille et Jean-Baptiste Dupuy, Longueuil 8 juin 1836, RAPQ (1945), 192-93. - Aussi, "J.-B. Dupuy à Mgr Lartigue", s.d., RAPF, 1 (1839): 24; 25 . La relation ainsi rédigée par M. Dupuy constitue l'une de nos principales sources. Elle est d'un style très simple et un peu impersonnelle de ton. L'auteur qui évite d'ailleurs de se mettre directement en cause, se dit anxieux de mettre beaucoup d'exactitude dans son récit afin d'être plus utile aux missionnaires de l'avenir. Nous utiljsons cette relation, non dans son texte original que nous ne connaissons pas, mais à travers la copie que nous en a donnée le Rapport de l'Association pour la Propagation de la Foi, 1 (1839): 25-23. 


\begin{abstract}
gens intelligents l'abrégé de la doctrine chrétienne pour servir de catéchistes capables d'instruire les autres et de baptiser au besoin; 3o. le baptême ne sera guère donné qu'aux moribonds, suivant la doctrine de saint Liguori; 4o. les missionnaires auront sur les sauvages d'au delà du township de Hull les pouvoirs spirituels que leur attribue le mandement de 1793, ainsi que ceux des archiprêtres et des curés concernant les mariages en particulier; 5o. ils s'informeront des principaux vices des sauvages et si les blancs leur vendent de l'eau-de-vie; 6o. ils apprendront l'état des sauvages, leur nombre, leur langue leurs causes, leurs rapports avec la Compagnie de la Baie d'Hudson; ils tiendront aussi un journal de leur mission, et ils transmettront les noms de ceux qu'ils auront choisis pour baptiser, marier et catéchiser ${ }^{18}$.
\end{abstract}

Donc le petit bateau à vapeur ${ }^{19}$, qui, tôt le 20 juin, quittait Lachine ayant à son bord M. Dupuy, arriva en vue de la mission $\mathrm{du}$ Lac à deux heures et demie de l'après-midi. Mr de Bellefeuille [ ou simplement Mr Bellefeuille, comme s'exprime la Relation d'un voyage $^{20}$ ] - Monsieur de Bellefeuille y monte et, tout de go, les deux missionnaires filent sur Carillon qu'ils atteignent environ deux heures plus tard. Ils suivent alors en berge le canal du LongSault pour reprendre le vapeur dès le lendemain. Et toute cette journée du 21 juin eut pour les voyageurs quelque chose d'une croisière sur l'Outawais que les beautés de nature parsemées sur les deux rives du Haut et du Bas-Canada rendent si grandioses: Grenville, Caledonia avec ses eaux minérales, la Petite Nation protégée par son sanctuaire de Notre-Dame de Bonsecours, la Rivière Rideau tourmentée par ses rapides et ses chutes, puis enfin la naissante et déjà coquette Bytown.

La canal Rideau avec ses "neuf écluses placées en amphithéâtre" 21 force l'admiration des voyageurs. Jeter un rapide coup d'œil sur Bytown est tout ce que peuvent faire ceux-ci, qui, sans tarder, doivent gagner Aylmer Place en "stage". Dix lieues les tiennent encore loin des belles chutes du Lac des Chats où ils vont

18. "Mgr Lartigue a MM. Charles de Bellefeuille et Jean-Baptiste Dupuy," Longueuil, 8 juin 1836, RAPQ (1944-45), 192-93.

19. J.-B. Dupuy, "Journal d'un voyage", RAPF, 1 (1839): 25.

20. Ibid., 25, 28, 298 .

21. On sait que le canal Ridesu fut achevé en 1832, L. Le Jeune, Dictionnaire gentral, 2: 392. 
devoir dire adieu au "steamboat", au "stage" et à la berge pour s'accomoder tant bien que mal des grands canots qu'avec courtoisie, du reste ${ }^{22}$, la Compagnie de la baie d'Hudson va mettre à leur disposition pour le reste du voyage. Or pour rencontrer les indiens du Lac Témiskaming et tenir mission à leur profit, il faut faire diligence, les Témiskamings venant vendre "leurs pelleteries aux traiteurs le 1 juin et le 25 juillet"'23.

Un contretemps attendait M. de Bellefeuille. La dite Compagnie ne disposait, au Poste des Chats, d'aucun canot en état de faire le voyage. M. McTavish, le bourgeois du poste, laissait toutefois espérer que Fort Coulonge en pourrait posséder un qui donnât satisfaction. Mais aller à Fort Coulonge et en revenir ne signifiait pas moins de cinquante lieues à parcourir le plus rapidement possible sur un frêle canot d'écorce d'environ deux brasses de long. Il fut décidé que M. Dupuy, plus jeune et de santé plus robuste, ferait le dur voyage avec les rameurs. Le texte même de la relation écrite par M. Dupuy rend un son qu'il importe d'entendre pour avoir une juste idée des faits:

En deux jours nous atteignîmes le Fort Coulonge... Il nous fallut pourtant faire deux portages et un détour assez long pour éviter un violent rapide d'une lieue qu'il y aurait eu à la tête des Chats: bientôt nous laissâmes derrière nous le Lac des Chats que viennent grossir les Rivières Mississipi, Madawaska et Bonnechère, que nous apercevions sur le $\mathrm{H}$. Canada: nous entrâmes dans les chenaux; puis nous fîmes le portage du Fort, qui a bien un mille de long. Le lendemain nous franchîmes sans peine les portages des Sables, de la Montagne, d'Angis, qui n'ont guère qu'un arpent ou deux, et celui du Grand Calumet, formée par une branche de l'Ottawa, appelée Rocher Fendu, qui se sépare de la Grande Rivière à la Passe, et s'y réduit au portage des Sables. Cette Ile a environ 8 lieues de long: le sol en paraît bon; elle est déjà habitée par un

22. C'est M. Dupuy qui s'exprime ainsi touchant l'attitude des bourgeois de la Compagnie de la baif d'Hudson: "Nous manquerions à la reconnaissance si nous passions sous silence l'ex sellente réception que nous eûmes de $\mathrm{Mr}$ O'Neil, bourgeoys des cages. Etabli à l'entrée de la Rivière Bonne Chère... Mr Brown nous reçut au Fort Coulonge d'une manière à faire honneur à la religion catholique dont il fait profession, et à l'honorable Compagnie de la Baie d'Hudson, qu'il servait en qualité de commis. Canots vivres et toutes autres choses nécessaires au voyage nous furent fournis avec un empressement sans égal." J -B Dupuy, "Journal du voyage", RAPF., 1 (1839): 29.

23. Ibid., 44. 
grand nombre de catholiques, qui ont pensé à y bâtir une chapelle: mais malheureusement ils n'ont pu réussir. Leur éloignement de tout prêtre les expose à perdre les sentiments religieux et à mourir sans sacrements. Tout en déplorant leur triste sort, nous poursuivîmes notre route jusqu'au Fort Coulonge où nous arrivâmes vers les 5 heures du soir ${ }^{24}$.

M. Dupuy était ainsi parti le 24 "de grand matin". Les deux missionnaires ne devaient être de nouveau réunis que dans la soirée du 30, soit sept jours plus tard. Qu'importe, leur industrie saurait empêcher que cet isolement ne se muât en oisiveté. M. de Bellefeuille va passer le dimanche à Fitz-Roy (Haut-Canada): "où, note le Journal d'un voyage, il $\mathrm{y}$ a bon nombre de catholiques [ vraisemblablement des gens de chantiers] - qui sont dans le plus grand besoin de secours religieux"25. De son côté, M. Dupuy revenant de Fort Coulonge avec le canot désiré, fait halte à La Passe, et charge ses rameurs d'aller seuls quérir $M$. de Bellefeuille. Pendant ce temps il réunit les enfants des canadiens tant du Haut que du Bas-Canada:

Il lui parut que ce quartier était habité par 250 catholiques dispersés sur un espace d'environ 6 lieues de long. Les journées des 28,29 et 30 qu'il passa à catéchiser de pauvres enfans - [ sic ] - en attendant l'arrivée de $\mathrm{Mr}$ de Bellefeuille, furent des journées d'une vraie jouissance. Quoiqu'il y ait un égal mórite à faire connaître J.C. en quelque lieu que ce soit, l'on eprouve pourtant, ce semble, une consolation indéfinissable à le faire dans des lieux où ce bon maître est moins connu ${ }^{26}$.

Tard dans la journée du 1er juillet, ils arrivèrent à Fort Coulonge où devait s'effectuer la première rencontre des missionnaires avec

24. Ibid., 28-29.

25. Ibid., 30. Dans le même RAPF l'auteur du "Coup d'œil sur les missions du diocèse de Montréal", - Mgr Bourget, ou peut-être l'abbé Truteau, - prend a son compte le jugement de $M$. Brady, l'un des prêtres qui furent chargés de visiter ces catholiques: "Il devient indispensable, prétend celui-ci, de visiter fréquemment ces quartiers; sans quoi nos catholiques finiront par n'avoir plus de religion: car n'ayant ni Ecoles, ni Eglises et vivant au milieu des Protestants, qui nagent dans l'abondance et ont de jolis établissements pour leur culte et leur enseignement, ils sont exposés a la dangereuse tentation de prêter l'oreille à leurs discours. Les catholiques de ce Township - [ il s'agit de celui de Grenville ] — sont très dispersés... Les Ministres Méthodistes y exercent leur zèle, et y distribuent à pleines mains leur bibles falsifiées et leur Tracts.", 6-7.

26. Ibid., 30. 
un groupe de sauvages de quelque importance. Lorsque en 1832, Mgr Lartigue avait voulu envoyer le sulpicien Flavien Durocher dans le nord de l'Outawais, c'était "pour répondre, affirmait le prélat, aux ardents désirs des sauvages payens." ${ }^{27}$ L'attitude prise par les indiens de Fort Coulonge à l'égard des deux missionnaires confirme cette affirmation. A l'arrivée de ceux-ci, trente sauvages environ, dispersés dans les îles environnantes et qui s'occupaient a fabriquer des canots, viennent offrir leurs hommages de façon fruste mais émouvante. M. de Bellefeuille saisit l'occasion qui s'offre d'instruire ces enfants de la nature. Ceux-ci manifestent la volonté de l'entendre encore et de le garder avec eux. Mais il y a urgence de partir dès le lendemain, et sur la promesse de séjourner plus longuement au retour, les missionnaires rompus de fatigue prennent congé.

La nuit qui suivit fut un échec pour ceux-ci. Le chroniqueur note plaisamment que "des millions de maringouins avides apparemment de sang étranger se jettent sur nous avec tant d'acharnement que nous ne pouvons clore l'œuil"'28. Il fallut opter. M. de Bellefeuille choisit le parti de l'héroīsme et décida d'attendre l'aurore, armé de pied en cap, comme les preux du Moyen âge, c'est-à-dire tout habillé, son chapeau sur la tête et le visage dignement voilé. La décision de M. Dupuy fut différente - avait-il l'âme veule? - . Il préféra risquer l'asphyxie et "ne vit pas d'autre moyen d'échapper à la lancette de ces habiles saigneurs, que de s'enfermer dans ses couvertes [ sic ] - "préférant mourir de chaleur que de sentir des millions de sangsues ailées" 29 .

Tard dans la soirée du 3 juillet les deux missionnaires arrivaient au Fort des Petites Allumettes, appelé aussi Fort William du nom d'un ancien commis de la Compagnie de la Baie d'Hudson. L'établissement n'en imposait guère: "une couple de maisons, dit la relation, avec quelques hangard, sans aucuns retranchemens" ${ }^{30}$. C'était là cependant une nouvelle étape qui rapprochait de Témiskaming; et puis, pour la première fois, $M$. de Bellefeuille et son compagnon allaient pouvoir "missionner" dans les formes chez un groupe

27. "Mgr Lartigue à Mgr Panet", Montréal, 12 mai 1832, RAPQ (1943), 145.

28. "Journal d'un voyage", RAPF, 1 (1839): 32-33.

29. Ibid., 32-33.

30. Ibid., 32-33. 
d'indiens de relative importance. Ils étaient environ cinquante. D'abord timides mais attentifs, ils cèdent très vite à leur intuition et au désir qu'ils ont de connaître les hommes de Dieu. Gagnés par l'attitude paternelle de ceux-ci ils assistent à la messe que célèbre M. Dupuy pendant que $M$. de Bellefeuille secondé par deux jeunes indiens originaires du Lac des Deux-Montagnes, chantent des cantiques.

Les indiens étaient gagnés. Anxieux de "connaître une religion que déjà ils avaient appris à aimer" 31 , ils écoutent $M$. de Bellefeuille, qui leur fit un discours "analogue à leurs besoins", avec ce résultat que 21 enfants au-dessous de sept ans furent présentés au baptême. Les pères et mères exultaient. Toute la journée s'était passée à catéchiser, baptiser et confesser. Ce dut être un moment de joie profonde, car M. Dupuy formule ce jugement par manière de conclusion: "on se croit aux premiers siècles de l'Église. ${ }^{32}$ "

Dans la journée du 4 juillet les missionnaires quittaient le Fort des Allumettes pour n'arriver que le 14 à Témiskaming. Ils avaient donc en perspective dix lourdes journées de navigation ${ }^{33}$, coupées seulement d'une très courte halte au poste Matawan. A une lieue et demie plus haut que le fort William qu'ils venaient de quitter, s'ouvrait la Rivière Creuse, qui n'est d'ailleurs qu'une modification de l'Outawais. Celle-ci prend ici une profondeur considérable et sa largeur d'une demi-lieue reste constante sur une distance de douze lieues. Le texte de la relation reflète ici l'admiration ou l'ennui profond qu'éprouvent les voyageurs selon la qualité du panorama qu'ils ont sous les yeux:

Cette partie de la Grand Rivière - [l'Outawais ] est vraiment magnifique et parfaitement droite dans tout son cours de douze lieues. Les rivages sont variés par de jolies pointes et de beaux rochers. Elle est bordée, au nord de montagnes, entre lesquelles on remarque celle appelée l'Oiseau, à une lieue de l'embouchure, laquelle - [ la montagne ] - est

31. Ibid., 34 .

32. Ibid., 34.

33. Il convient de rappeler ici que, dans leur navigation, les voyageurs avaient ou a faire portage ou ramer contre le courant. La ligne de la hauteur des terres est encore loin, qui est située au nord du Lac Témiskaming. Voir à ce sujet "Carte de la Nouvelle France pour servir à l'étude de l'histoire du Canada au XVIIe et XVIIIe sieccle," L. Lejeune, Dictionnaire general, 2:358. M. de Bellefeuille n'atteindra et ne dépassera cette ligne que l'année suivante dans la mission qu'il fera au Lac Abitibi. 
coupée perpendiculairement, et s'élève au-dessus de l'eau au moins 300 pieds... Depuis l'entrée de la Rivière creuse jusqu'à Témiskaming il n'y a plus d'habitans. On ne trouve que trois maisons de chantiers, et deux chaumières, qui appartiennent à la Compagnie, l'une à l'entrée de la rivière du Moine et l'autre à Matawan ${ }^{34}$.

La topographie des lieux soigneusement observée et décrite par M. Dupuy permet de suivre pas à pas les voyageurs. Ils franchissent tout d'abord le Portage des Joachim situé un peu plus haut que la Rivière Creuse. Puis ils doivent traverser l'embouchure de la Rivière du Moine qu'ils aperçoivent à leur droite, sur la rive nord de l'Outawais. Une nouvelle difficulté à vaincre s'offre a eux: le difficile portage de la "Roche à Capitaine", long, il est vrai, de quelque quinze arpents seulement, mais quand même tristement fameux pour les dangers de mort qu'il présente pour le voyageur téméraire.

Le 9 juillet, ils arrivaient au minuscule poste de Matawan, situé sur la pointe que forme avec l'Outawais, la petite rivière Matawan par où l'on gagne le lac Nipissing. Cette halte de quelques heures eut quelque chose d'une oasis pour les deux prêtres. Tout d'abord, un peu avant d'arriver au poste, $M$. de Bellefeuille fait la rencontre de deux familles qui s'hébergent dans une maison de chantier et pour qui il a eu autrefois des bontés de père à la mission du Lac. Joie profonde de ces pauvres gens et de celui-ci, qui sitôt arrivé au poste commence bien vite d'instruire et de confesser les sauvages pendant que M. Dupuy remonte jusqu'à la fourche de la rivière Matawan. Les gens de chantiers sont convoqués pour la messe du lendemain à laquelle, note la relation, "assistèrent trente à quarante personnes". Et le chroniqueur d'ajouter avec quelque lyrisme: "Providence admirable qui réunit autant de monde dans un lieu si écarté, où la victime pure et sans tache, qui, selon le Prophète, doit être offerte du Levant au Couchant n'a jamais été immolée. Les airs et les forêts retentissaient des louanges du Seigneur ${ }^{35}$."

Dès 11 heures de la même journée, 10 juillet, le grand canot de la Compagnie de la baie d'Hudson entreprenait la dernière étape de son voyage; trente lieues séparaient encore les missionnaires

34. "Journal d'un voyage", RAPF, 1 (1839): 35.

35. Ibid., 37. 
de Témiskaming. Le rapide des Caves et des Chaudières fut la première curiosité de nature qui attira l'attention des voyageurs. M. Dupuy, dont l'âme paraît avoir été très accessible au pittoresque et au grandiose, fait de pertage une description qui vaut d'être citée:

Nous fîmes un peu plus d'une lieue; et nous nous trouvâmes au Rapide des Caves. Ce rapide est extrêmement violent et rempli de remous, ou tournoiements d'eau, causés par la rudesse et l'inégalité du rocher sur lequel il coule. Il y a dans ce portage des Caves et des Chaudières. Les Caves ne sont autrechose que des lits de rochers brisés et soulevés probablement par quelque tremblement de terre; et qui forment des espèces de voûtes très irrégulières, garnies d'une mousse fort épaisse, et dans lesquelles on trouve encore de la glace au mois de juillet. Les Chaudières sont des espèces de puits, creusés perpendiculairement dans le roc. Elles sont d'une forme ronde, et parfaitement régulière comme si elles eussent été faites autour - [ sic ] -. Il y en a un grand nombre et de difféententes dimensions, depuis un jusqu'à six et sept pieds de diamètre; et depuis un jusqu'à six ou sept pieds de profondeur. Ces puits ou chaudières sont évidemment formés par des roches plus ou moins grosses qui, entraînées et agitées ensuite par des remous, tournent continuellement à la même place au fond de l'eau; et leur frottement contre le roc y creuse des Chaudières. Dans la saison des basses-eaux, ces lieux étant à sec et découverts, les voyageurs ont la facilité d'observer ce singulier ouvrage de la nature" 36 .

Le portage des Erables et le rapide du Long Sault une fois franchis dans la journée des 11 et 12 juillet, les voyageurs entrèrent tout aussitôt dans les eaux du Lac Témiskaming. Toutefois, il leur restait encore quinze lieues à faire pour parvenir au poste de la Compagnie; c'était là l'endroit jugé propice pour y organiser la mission. Ce n'est donc que le 14, à quatre heures de l'après-midi, que les deux missionnaires de Jésus-Christ prirent un premier contact avec leur future chrétienté. 150 lieues les séparaient de Montréal. L'exclamation "Hourra, Témiskaming!" poussée par les voyageurs arrivant en vue du poste, donne à penser qu'ils crurent entrer dans la Terre promise. Cette joie apostolique se conçoit sans peine.

Malgré son importance pour la traite des fourrures, le poste de la Compagnie à Témiskaming n'était pas matériellement considérable:

36. Ibid., 38. 
"quatre maisons et quelques bâtimens [ sic ] ${ }^{37}$." Cependant la réception y fut chaude ${ }^{38}$. On hissa le pavillon; tous les personnages officiels du poste vinrent souhaiter la bienvenue aux missionnaires: c'était M. Hardesty, qui agissait comme bourgeois du fort en l'absence de M. Cameron; M. Mc Auly, le commis; enfin M. McKay qui faisait fonction d'interprète dans les tractations entre blancs et Indiens. On pouvait en conséquence augurer favorablement de l'attitude de la Compagnie dans l'œuvre d'évangélisation projetée. Dès le 15 au matin, les deux prêtres s'attaquèrent à la besogne.

Pendant que M. de Bellefeuille, après avoir réuni "dans le haut d'un grand hangar" les quelques sauvages demeurés au poste, s'occupait de leur donner les premières instructions, M. Dupuy jouait le personnage de Marthe, réglant les détails matériels de l'entreprise, préparant un oratoire où l'on pût célébrer la messe décemment, veillant à ce qu'on préparât le bois de la croix que sur les ordres de Mgr Lartigue on devait planter a Témiskaming avant la fin des exercices spirituels ${ }^{39}$. Il visita aussi les malades et distribua aux nécessiteux les secours sans lesquels ils ne pourraient séjourner au poste les 13 jours que durerait la mission. Enfin il s'occupa de préparer l'érection de la chapelle en faisant équarrir par les rameurs une partie du bois nécessaire ${ }^{40}$.

Cependant, chez les sauvages qui, à l'approche des missionnaires, avaient pris la fuite un peu comme des enfants, la défiance, les préventions tombèrent et:

bientôt l'on vit arriver des fonds du Lac des canots chargés de femmes et d'enfans. Tous montrèrent une ardeur et un empressement incroyables à profiter des instructions de Mr.

37. Ibid., 40. On sait d'autre part que les sauvages faisant la traite des fourrures au poste de Témiskaming étaient au nombre de 220, Ibid., 43.

38. Cela est vrai tout au moins pour ce qui est de la Compagnie. Car, ainsi que le note M. Dupuy "sur la nouvelle de notre arrivée, plusieurs - [ sauvages ] - qui résidaient ordinairement au poste s'étaient enfuis. Il paraít que le bruit s'était ró pandu parmi eux que les hommes de la priere venaient s'emparer du poste pour punir les méchans." Ibid., 40.

39. Mgr Lartigue, "Instructions à MM. Charles-Louis de Bellefeuille et JeanBaptiste Dupuy, missionnaires au Lac Témiskaming," Longueuil, 8 juin 1836, RAPQ. (1945) 192-93.

40. La "Relation d'un voyage" note que l'interprète mentionné plus haut "se chargea de faire tirer aux premieres neiges tout le bois du comble." p. 51 . 
Bellefeuille. Plusieurs joignant la facilité à une grande application purent, pendant les 13 jours que nous passâmes à Tómiskaming, apprendre les principales vérités de la religion, avec le Pater, l'Ave et le Credo en leur langue: si bien qu'ils furent jugés capables de recevoir le baptême ${ }^{41}$.

Journées remplies! Une première messe à six heures du matin, que célébrait M. de Bellefeuille pour les employés de la Compagnie. Puis entre huit et neuf heures, M. Dupuy célébrait à son tour, mais au profit des Témiskamings eux-mêmes, pendant que M. de Bellefeuille récitait des prières ou chantait des cantiques, le tout en langue algonquine. Après quoi se faisait l'instruction suivie de l'administration du baptême s'il y avait lieu. La substance de la doctrine exposée n'était autre que le plan providentiel sur l'homme: l'existence de Dieu, la création de l'univers, l'au delà éternel de la vie humaine, la faute originelle, l'œuvre du Rédempteur, enfin les récompenses éternelles pour ceux qui sont fideles au Sauveur ou bien punitions de ceux qui trahissent. Or, il faut l'observer, toutes ces choses étaient dites à des esprits manichéens ${ }^{42}$, qui admettaient à l'origine de toutes choses, l'existence de deux êtres, l'un cause du bien, l'autre, fauteur du mal. Et cependant, note M. Dupuy, on voyait se peindre spontanément sur les figures des sentiments d'approbation et de bonheur, "comme s'ils eussent fait la découverte de quelques mystères qu'ils auraient cherché depuis longtemps" ${ }^{43}$. Comme quoi les mystères chrétiens conservent, en dépit de leur transcendance et de leur obscurité, des affinités lointaines avec l'esprit humain. Détail typique "ceux qui ne pouvaient trouver place dans la tente, qui était bientôt encombrée... demeuraient en dehors et se passaient au moins la tête par dessous la toile, se tenant dans cette attitude tout le temps que durait l'instruction ${ }^{44}$."

Ces êtres qui naissaient ainsi à la foi chrétienne, qu'étaient-ils donc? Des âmes ayant, en somme, plus besoin d'instruction que d'apologétique; des êtres en général pacifiques, patients, tranquilles jusqu'à la timidité. Peu de polygames parmi eux. Et encore, ceux
41. "Journal d'un voyage", RAPF, 1 (1839): 41.
42. Ibid., 43.
43. Ibid., 42.
44. Ibid., 43. 
dont la condition de vie est telle sont-ils regardés avec mépris. L'impureté n'est pas la faute dominante, quoique, note le Journal d'un voyage, "le péché des blancs avec les femmes sauvages soit connu ici comme ailleurs" ${ }^{45}$. Mais en revanche l'abus des boissons alcooliques, l'ivrognerie abêtit ces peuplades de façon inoule. Point de culte public parmi eux; tout au plus, et rarement, des sacrifices particuliers. Enfin aucun jongleur; aucun chef dont la fonction reconnue soit celle d'offrir le sacrifice. Et, - telle fut la conclusion à laquelle en arrivèrent les deux missionnaires, - peu de chose les tenait loin du Christ. Si, à raison de leur inconstance native, leur persévérance dans la foi chrétienne restait problématique, en revanche leur conversion s'avérait des plus faciles.

La volonté de Mgr Lartigue, on se le rappelle, était que, par manière de prise de possession, une croix fât érigée sur l'emplacement de la future chapelle ${ }^{46}$. On procéda à la cérémonie dans l'aprèsmidi du 19 juillet et l'événement marqua. Devant les Indiens réunis pour l'instruction de quatre heures, $M$. de Bellefeuille fit porter les réflexions sur la Croix de Jésus, sur les raisons que l'on avait de l'ériger et la manière dont il faudrait la vénérer. Puis la jeune chrétienté de Témiskaming se mit en marche vers l'endroit choisi, la croix ouvrant la procession. Après que les litanies eurent été chantées en langue algonquine, toujours sur les instructions de l'évêque de Montréal, Saint-Adalbert, évêque de Prague et martyr, fut proclamé patron de la mission ${ }^{47}$. Et l'on procéda à l'érection au milieu d'un silence général. L'hymne $O$ crux Ave mit fin à la cérémonie. A défaut d'une chapelle érigée dans les formes, la chrétienté naissante de Témiskaming possédait ce qui pouvait en tenir lieu provisoirement: la croix du chemin.

Les exercices spirituels se poursuivirent jusqu'au 27 juillet. A cette date, ils duraient depuis 13 jours. Les provisions s'épuisant, M. de Bellefeuille n'en pouvant plus de fatigues, il fallut songer au

45. Ibid., 46.

46. Mgr Lartigue, "Instructions à MM. Charles-Louis de Bellefeuille et JeanBaptiste Dupuy, missionnaires au Lac Témiskaming," Longueuil, 8 juin 1836, RAPQ, (1945) 192-93.

47. Nous ignorons les raisons pour lesquelles Mgr Lartigue a tenu à donner, de préférence à tout autre, Saint-Adalbert comme patron à la chrétienté de Tómiskaming. 
retour. Le départ s'effectua le 27 à onze heures du matin, et l'attitude des employés de la Compagnie comme celle des Indiens euxmêmes rendirent la séparation émouvante: "Le rivage, note mélancoliquement M. Dupuy, était couvert de sauvages. Nous leur fîmes notre adieu... Il régnait partout un grand silence et plusieurs versaient des larmes"48. Le retour, marqué de plusieurs arrêts, prit trois semaines aux voyageurs qui rentrèrent à Montréal le 16 août. Le bilan de la mission pour l'année 1836 pouvait être dressé comme suit: 56 jours d'absence; 300 lieues de canotage à travers lacs, rapides et portages; " 142 baptêmes (123 d'enfans et 19 adultes) 28 premières communions, et 4 mariages" ${ }^{49}$. L'apostolique aventure valait qu'on la tentât de nouveau.

\section{MISSION AU LAC ABITIBI \\ (7 juin -23 août 1837$)$}

C'était seul toutefois que M. de Bellefeuille devait entreprendre la randonnée de 1837. En effet, on était à l'époque où à l'instigation du clergé et par réaction contre "L'Institution royale pour l'avancement des sciences", le Bas-Canada se couvrait d'écoles latines. Chambly avait la sienne, qu'avait fondée Messire Pierre-Marie Mignault, curé de la paroisse ${ }^{50}$. M. Jean-Baptiste Dupuy, après avoir donc été directeur de ladite école pour la journée du 10 octobre 1836 devenait dès le lendemain curé de la nouvelle paroisse de Saint-Anne-sur-Yamaska ${ }^{51}$. Il y demeurerait quatre ans (183640). Donc, lorsque M. de Bellefeuille quitta Lachine, le 7 juin 1837, pour une mission qui s'avérait plus pénible et plus lointaine que la précédente, il ne jouissait pas d'une santé meilleure ${ }^{52}$ et, circonstance aggravante, il ne pouvait compter que sur lui-même. Ceci ajoute, pensons-nous, à son mérite ${ }^{53}$.

48. "Journal d'un voyage", RAPF, 1 (1839): 51.

49. Ibid., 53.

50. Y. Charron, "Le Collège Classique de Saint Pierre à Chambly", La Société canadienne d'Histoire de l'Eglise catholique, 13 (1945-46): 1938.

51. "Mgr Lartigue à M [ J.-B. ] Dupuy, directeur du Collège de Chambly", 10 octobre 1836, RAPQ (1944-45), 211.

52. [ Mgr Bourget, probablement] "Avant-Propos", RAPF, 2 (1840): 9.

53. A travers les écrasants labeurs de la mission et du voyage, il devra aussi griffonner quelques notes, desquelles il tirera au retour le texte de la relation qui 
Au cours de son voyage le missionnaire fit quatre haltes principales. A l'aller, Témịskaming qui le retint deux semaines et Abitibi où il s'attarda neuf jours. Au retour, de nouveau Témiskaming et enfin le Fort des Allumettes, deux postes auxquels M. de Bellefeuille consacra respectivement six et dix jours. Ce fut dans un grand Canot-du-Nord mis à sa disposition par la Compagnie de la Baie d'Hudson, qu'il quitta Lachine avec quatre rameurs. L'embarcation en était une de huit ou neuf rames ${ }^{54}$, et même elle pouvait porter voile. Après avoir effectué un premier portage, - en voiture, celuilà, - au rapide de Sainte-Anne-de-Bellevue, les voyageurs essuient une si dure tempête sur le Lac des Deux-Montagnes que leur vie est en danger ${ }^{55}$. Ce n'est que le surlendemain, 9 juin, que M. de Belle-

constitue notre source principale pour la mission de 1837. Nous avons en mains, - non l'original, - mais la copie qu'en a publiée le Rapport de l'Association pour la Propagation de la Foi, 2 (1839): 16-72. En lisant 1" "Avant-propos" du RAPF, 2 (1840): 9, le lecteur pourra juger de l'état et de l'intégrité du texte dont nous nous servons: "Nous nous faisons un devoir de donner aux associés, y est-il affirmé, la relation de sa seconde mission - [ celle de 1837 ] - telle qu'il l'a rédigée. Nous le laissons parler lui-même avec ce langage simple et touchant, qui a toujours été celui des hommes apostoliques. Nous nous serions fait scrupule de priver la postérité de ce monument de la piété et du zèle de cet excellent missionnaire. Si nous avons fait quelques changements, c'est parce que lui-même se proposait de le faire, lorsqu'il eut. consenti à permettre l'impression de ce journal." De cette relation, nous avons cité entièrement le titre, pourtant très long ainsi que le sigle, sous la note 9 . Ce récit de M. de Bellefeuille est plus élaboré que le "Journal d'un voyage", et cependant la lecture en reste captivante, car $M$. de Bellefeuille l'a rédigé dans un style alerte, vivant, où passe toute l'attachante personnalité de ce prêtre très humain et non moins apostolique.

Il convient enfin d'observer que la rédaction définitive fut faite a Montréal, entre septembre et décembre 1837 , alors que les troubles politiques et militaires de l'époque jetaient l'anxiété dans les esprits. C'est là du reste ce qu'affirme l'auteur de la relation dans une lettre adressée à Mgr Signay: "Monseigneur, ayant apris que Votre Grandeur, avait accueilli et lu avec bien du plaisir le journal rédigé par $\mathbf{M}$. Dupuy relativement à la mission que nous fîmes ensemble l'année dernière au Lac Témiskaming, je me suis déterminé à Lui présenter encore la relation d'une seconde mission que $j^{\prime}$ 'ai faite il y a quelques mois, dans le même lieu et au delà, jusqu'au lac Abbitibbi qui fait partie de son diocèse et du district de Monseigneur de Juliopolis... J'espère aussi que Votre Grandeur, en lisant ce journal, voudra bien excuser les fautes de liaison et de style qui s'y trouvent, car, comme Elle aura souvent l'occasion de le remarquer il se ressent beaucoup du trouble et du désordre causés par les agitations de la guerre civile qui nous afflige dans ce temps malheureux où l'on me presse de l'écrire. D'ailleurs il m'a fallu pour l'écrire, tout tirer de ma pauvre mémoire, n'ayant pu faire presque aucunes notes - [ sic ] - parce que les occupations continuelles de la mission me laissaient à peine le temps de vaquer à mes exercices spirituels, et d'enregistrer mes actes de baptêmes, mariages, etc.", cité dans Pierre Trudelle, L'Abitibi d'autrefois, d'hier et d'aujourd'hui (Amos, Qué., 1937), 30-31.

54. Il faut entendre avirons ou perches.

55. C. de Bellefeuille, "Relation d'une mission", RAPF, 2 (1840): 8-19. 
feuille peut faire une brève apparition à la mission du Lac, et complète son équipage en louant les services d'un guide et d'un pilote ${ }^{56}$.

Le 10 juin, une rencontre heureuse. Alors que les voyageurs, après avoir dépassé Chatam, sont "campés pour coucher" 57 sur l'Outawais, dix-huit canots passent au large. Ce sont des sauvages qui viennent de leurs territoires de chasse et débouchent par la Rivière du Lièvre. Ils se rendent à la mission du Lac. Parmi les Indiens, M. de Bellefeuille compte de vieilles et chères connaissances. Les jours suivants ramènent le panorama de l'année précédente: Bytown, le rapide et le Lac des Chênes, le rapide et le Lac des Chats, enfin le portage de la Montagne où le missionnaire a le bonheur de baptiser en la nuit du 14 juin, cinq des enfants de J. Worrill, un fermier américain établi à proximité. La nuit du 15 juin fit époque dans l'esprit du chroniqueur, qui écrit ces mots: "Nous allâmes coucher au Crand Marais, où je passai une partie de la nuit à confesser et l'autre partie de la nuit à me défendre contre les cousins, les moustiques et les brulots dont la maison était pleine".

Couchant parfois sous la tente, parfois aussi bénéficiant de l'hospitalité offerte par les fermiers ou les gens de chantiers, les voyageurs poursuivent leur pénible randonnée. Le 17 juin ils revoient le portage de la Culbute, dans l'île des Allumettes; deux jours plus tard, ils font halte au pied du rapide la Roche-capitaine. C'est là que des sauvages du Lac, maintenant établis dans la région et cultivant "patates", blé d'inde et citrouilles"59, le pressent de leur dire la messe. Il n'est que de lire le texte de la Relation d'une mission pour comprendre que $M$. de Bellefeuille eut alors un mouvement de joie profonde; en somme le bonheur de l'homme de Dieu lorsqu'il a le sentiment vécu de sa médiation.

56. On l'appellera parfois le gouvernail, Ibid., 70. De même "Précis dela relation", RAPF, 2 (1840): 73.

57. C. de Bellefeuille, "Relation d'une mission", RAPF, 2 (1840): 20.

58. Ibid., 24. On ne lira pas sans intérêt la description finement ironique du banquet-gueuleton qui suivit, et au cours duquel fraternisèrent très démocratiquement les voyageurs, les Indiens et même "les quinze ou seize chiens de la cabane", Ibid., 27. M. de Bellefeuille s'y révèle capable d'un humour qu'il fait plaisir de trouver chez cet homme par ailleurs malade et quelque peu porté, semble-t-il, à la mélancolie.

59. Ibid., 26. 
Le 20 juin, le missionnaire passait le rapide de l'Eveillé dans lequel, le mois précédent, avait sinistrement péri un traiteur. Le poste Matawan, le portage des Caves, le rapide du Long-Sault furent les étapes suivantes; et enfin, "le 24, après 16 jours de navigation, note M. de Bellefeuille, nous entrâmes dans le lac Témiskaming, que nous passâmes par un bon vent à pleine voile" 60 . Réception chaleureuse. Le pavillon du fort flotte au vent; la pointe est couverte de cabanes et presque tous les sauvages se trouvent réunis. Le bourgeois, M. Cameron, les commis, les hommes, les femmes, les enfants, catholiques comme protestants et infidèles, souhaitent la bienvenue aux arrivants, si bien que $M$. de Bellefeuille doit abandonner son premier dessein d'aller d'abord au poste d'Abitibi ou au Grand Lac Victoria, et décide de faire mission à Témiskaming sans plus tarder.

Le soir même de l'arrivée, les exercices commencent "dans le haut du Grand Hangar ${ }^{61}$. Les nouvelles sont bonnes: au témoignage de M. Cameron, la conduite des sauvages attachés au poste a été excellente; un certain nombre d'Indiens, encore infidèles et qui s'étaient trouvés absents l'an dernier, se présentent d'eux-mêmes aux exercices de la mission; des conversions d'importance s'opèrent, telle, par exemple, celle d'un polygame, telle aussi celle d'Indainwé, un des sauvages les plus marquants du poste. Enfin des initiatives aussi touchantes qu'ingénieuses se produisent:

Un jour, ecrit M. de Bellefeuille, que, dans un moment de loisir, j'étais à converser avec le bourgeois et un commis, un jeune sauvage de 16 à 17 ans m'aborda modestement, et me présentant un morceau d'écorce de bouleau, qu'on pourrait appeler papier sauvage, il me dit en me montrant quelques marques qu'il avait tracées dessus: "tiens, mon père, regarde ce papier; voilà tout ce que je sais de la prière." C'était le Pater, l'Ave, le Credo, et les actes des vertus théologales qu'il y avait écrit à sa manière... le jeune homme avait tracé sur son papier, avec assez de régularité, des lignes plus ou moins longues suivant la longueur des paroles; autant de paroles autant de lignes"62.

60. Ibid., 31 .

61. Ibid., 32.

62. Ibid., 36. 
Quinze jours durant, le missionnaire s'occupa d'instruire, de baptiser, de confesser. Il s'occupa de construire aussi, puisqu'il employa ses hommes "à couper et à équarrir les bois nécessaires à la bâtisse - [ sic ] - d'une chapelle"63. Tout d'abord ce furent les tout-petits qui reçurent le baptême; puis les autres, ceux qui tout en ayant moins de sept ans, avaient néanmoins reçu un minimum d'instruction religieuse. C'était là, du reste, un stimulant pour les adultes eux-mêmes, les pères et mères surtout; c'était un profond motif de joie pour le prêtre qui voyait ainsi croître une nouvelle chrétienté. En particulier celui-ci semble être demeuré, plusieurs mois plus tard ${ }^{64}$, sous le charme de certaines manifestations de vie chrétienne qu'il décrit avec une pointe manifeste de poésie:

L'empressement de ces bonnes gens pour s'instruire était tel qu'ils ne m'auraient pas laissé le temps de dire mon office, ni même de manger ou de dormir; et véritablement, ils semblaient quelques fois oublier de satisfaire à ce besoin pour eux-mêmes. Car il m'est arrivé plusieurs fois en sortant à ma porte vers le milieu de la nuit, d'entendre tout autour de la pointe, dans presque chaque cabane, ces bonnes gens réciter leurs prières et s'instruire mutuellement; j'entendais dans toutes les directions, comme le bourdonnement ou l'agréable murmure d'abeilles bien occupées dans leurs ruches ${ }^{65}$.

Mais nécessité fait loi, et vers la fin de la quinzaine il apparut manifeste à M. de Bellefeuille qu'il faudrait émigrer et disperser le petit troupeau. Les sauvages commençaient à manquer de nourriture, car "le lac épuisé ne fournissait plus assez de poisson pour cette bande de 300" bouches ${ }^{66}$. Le poste du lac Abitibi allait donc devenir la deuxième grande étape du voyage, et désormais ce fut de ce côté que se tournèrent les préoccupations du missionnaire. Le 9 juillet, à onze heures du matin, il quittait donc Témiskaming, non toutefois, sans avoir célébré la messe et donné l'instruction. Son

63. Ibid., 42. Signay.

64. Voir la note 53, où nous avons cité la lettre de $\mathbf{M}$. de Bellefeuille à $\mathbf{M g r}$

65. C. de Bellefeuille, "Relation d'une mission", RAPF, 2 (1840): 35.

66. Nous restons un peu sceptiques devant cette raison alléguée par $M$. de Bellefeuille. La pénurie de vivres ne s'expliquerait-elle pas plutốt par l'impossibilité dans laquelle se trouvaient les Indiens d'aller pêcher au loin sur le lac tout en suivant les exercices de la mission? 
équipage comptait désormais dix hommes plus un nouveau guide, le pieux sauvage Wabimango, dont la présence était rendue nécessaire par la difficulté et l'inconnu des régions nouvelles à franchir. Ce serait une étape de cinq jours et demi, tout à la fois difficultueuse et dépourvue de pittoresque. Les voyageurs, qui paraissent s'y être ennuyés ferme à certains moments, ne rencontrèrent pas âme qui vive. Pas même d'animaux, à l'exception de deux petits lièvres "et leur mère que la femme de notre guide prit au collet pendant la nuit, et dont elle fit un bon repas ${ }^{67}$."

Mais d'un autre point de vue, ni l'imprévu ni les émotions ne manquèrent aux voyageurs. Ceux-ci sortirent du Lac Témiskaming par la rive nord-est, et se trouvèrent tout aussitôt devant la tâche énorme de franchir la série des Quinze Portages consécutifs ${ }^{68}$. Ils y mirent toute la journée du 10 juillet et une partie du lendemain; et si, d'une part, la hauteur des eaux leur permit de réduire a 13 le nombre des portages, en revanche, un peu en deçà du quatrième rapide, une roche pointue perfora largement le fond du canot qui commença de faire eau avec grand dommage pour le bagage et quelque danger pour les hommes.

Les Quinze Portages une fois franchis, on tomba dans un lac que la Relation n'identifie pas ${ }^{69}$. "Vers le tiers de la longueur de ce lac, note $M$. de Bellefeuille, notre guide nous fit observer à notre droite une Baie par où l'on prend le chemin qui conduit au poste appelé Grand Lac, où je pense aller l'année prochaine en revenant d'Abitibi. D'après tout ce que j'ai appris des gens de ce poste, un missionnaire y sera reçu à bras ouverts par une peuplade aussi considérable qu'à Témiskaming"70. La Grande Savanne dans laquelle entrèrent les voyageurs le 13 juillet et qui fait suite à la Rivière Ennuyante marqua le recommencement des misères pour ceux-ci. Sur la longueur de cinq arpents, ils durent avancer péniblement,

67. C. de Bellefeuille, "Relation d'une mission", RAPF, 2 (1840): 46.

68. Ces portages sont généralement distants d'un mille ou d'un quart de lieue, et il est alors nécessaire de porter canot et bagage à travers bois et montagnes.

69. Ce ne peut pas être le lac des Quinze, qui aurait éloigné les voyageurs de leur objectif.

70. C. de Bellefeuille, “Relation d'une mission", RAPF, 2 (1840): 48-49. 
ayant de l'eau et de la vase jusqu'aux genoux et se trouvant si embourbés par moments qu'ils redoutèrent l'enlisement.

Le lac Labyrinthe ${ }^{71}$ marqua un moment d'intérêt pour $M$. de Bellefeuille qui parle de celui-ci comme d'un joli lac de forme ronde et d'environ une demi-lieue de diamètre... environné presque entièrement de belles montagnes. Il signale encore, en bon géographe, qu'on arrive ici à la hauteur des terres, car tous les rapides désormais rencontrés coulent vers le nord ${ }^{72}$, vers la baie James. Tout ceci se passait le 13 juillet. Dès le lendemain, 14, vers les neuf heures du matin, l'équipage se trouvait à l'entrée du lac Abitibi, à proximité du poste que la compagnie de la Baie d'Hudson avait établi "sur l'extrémité d'une longue pointe et du côté est"'73. Le missionnaire qu'envoyait ainsi Mgr Lartigue aux infidèles de cette région se trouvait alors à quelque deux cents lieues de Montréal, et s'apprêtait à jeter dans cet autre milieu païen, mais bien disposé, les fondements d'une nouvelle chrétientét ${ }^{74}$.

Le poste Abitibi comptait deux maisons, dont celle du bourgeois qui était alors M. Fraser, deux petits hangars ou magasins contenant provisions, marchandises et pelleteries, enfin un autre hangar où l'on remisait les canots. Situé sur un immense et beau lac de 24 lieues de longueur, à proximité relative de Moose Factory ${ }^{75}$, il offrait de plus grandes ressources aux Indiens, qui pouvaient y vivre plus à l'aise qu'à Témiskaming ${ }^{76}$. Tel était le poste dans lequel $M$. de Bellefeuille allait passer huit jours dans une atmosphère d'abord trouble, mais qui irait s'améliorant par la suite. A l'arrivée des voyageurs, il ne se trouvait au poste qu'un petit nombre de sauvages.

71. "C'est là, remarque M. de Bellefeuille, à peu près la hauteur des terres; car tous les rapides que l'on rencontre ensuite coulent vers le Nord." Ibid., 50.

72. C'est là, du reste, une observation corroborée par ailleurs, L. Lejeune, Dictionnaire général, 2: 358.

73. C. de Bellefeuille, "Relation d'une mission", RAFF, 2 (1840): 51.

74. Il est à remarquer que le territoire d'Abitibi appartenait canoniquement au diocèse de Québec. Ainsi s'explique la lettre adressée à Mgr Signay par M. de Bellefeuille, voir note 53 .

75. Moose Factory situé au fond de la Baie James n'est qu'à six jours de rames d'Abitibi.

76. 'Les provisions, dit la "Relation d'une mission', y sont moins rares et moins chers. Les draps, les fers, la munition et beaucoup d'autres articles y sont meilleur marché qu à Montréal même." RAPF, 2 (1840): 63. 
Encore n'était-ce que des femmes attendant leurs hommes, qui d'une journée à l'autre devaient revenir du Fort Moose, en compagnie de M. Fraser et dans des canots chargés de provisions venues d'Angleterre. D'autres sauvages attendaient à distance, eux aussi, et dans la défiance, car un de leurs congénères venu de Témiskaming les avait assurés que le baptême donnait la mort à quiconque le recevait.

Madame Fraser offrit bien, il est vrai, une très honnête hospitalité; mais M. Paulson, le commis, ignorant des sentiments du bourgeois alors absent et désireux de ne point se compromettre, se cantonnait dans une correcte froideur. Tout cela, avec d'autres circonstances d'importance secondaire, exiguïté du local, mauvaise température, tout cela fit que, si $M$. de Bellefeuille eat écouté l'avis des siens, il aurait secoué "sur cette terre la poussière de ses pieds" pour retourner bien vite vers Témiskaming, qui dans le désenchantement de l'heure, faisait figure d'oasis. Mais M. de Bellefeuille avait décidé de tenir bon, et à la fin bien lui en prit. Arrivé le vendredi, 14 juillet, au matin, il avait commencé les exercices de la mission à peine quelques heures plus tard, et les avait continués avec un succès relatif jusqu'au lundi matin.

Ce fut alors qu'arriva de Moose Factory, M. Fraser, le bourgeois du poste, avec son cortège de sauvages qui montaient des canots chargés de provisions. "Dès son arrivée, note le missionnaire, il témoigna le plus grand plaisir de me voir chez lui, et me promit de m'aider de tout son pouvoir"78. Ce fut le début d'une ère meilleure. M. Paulson, le commis métis, changea d'attitude jusqu'à demander le baptême pour tous ses enfants, prétextant qu'il voulait élever ceux-ci dans le catholicisme. Bientôt, tous les sauvages du poste en agirent de même et présentèrent au baptême "tout ce qu'ils avaient d'enfants au-dessous de sept ans" 79.32 enfants et 2 adultes furent aussi faits chrétiens. Le mardi, 18 juillet, le "Grand être" prit pos-

77. Ibid., 52.

78. Ibid., 56. M. Fraser ne poussa-t-il pas tout de même le zèle jusqu à l'indiscr6tion. "Le 18 juillet, remarque le missionnaire, je fis la plantation de la croix... tout se fit avec ordre et édification; le bourgeovs y assista et força les plus obstinés A y assister aussi. Il en fit de même ensuite à tous les autres exercices; il ne permit jamais à personne de s'en absenter, pas même à ce guide qui montrait tant d'opposition pour le baptême". Ibid., 58 .

79. Ibid., 59-60. 
session officiellement du poste d'Abitibi: on procéda donc, comme à Témiskaming à l'érection d'une croix qui, à défaut de sanctuaire, polariserait la vie chrétienne des néophytes.

Toute la semaine du 16 juillet donna ainsi occasion au missionnaire de révéler à ces infidèles le nom et la loi de Dieu; ce qu'il fit avec cœur et en regrettant que les contrariétés du début l'eussent un peu paralysé. Les provisions de l'équipage s'épuisant, il donna le signal du départ, en les laissant tous quand même "dans de bien meilleures dispositions, et un certain nombre avec un bon commencement d'instruction pour espérer de les baptiser à la prochaine mission" 80 . Le 22 juillet, soit neuf jours après l'arrivée. M. de Bellefeuille prenait la route du retour, amenant avec lui deux enfants de M. Paulson, - un garçon de huit ans et "une très grande fille de dix-huit ans", - que le père voulait faire instruire et baptiser à la mission du Lac. Le retour à Témiskaming se fit en quatre jours; il en avait fallu presque six pour aller. Outre que les voyageurs étaient en pays désormais connu, on se rappelle aussi qu'à partir du Lac Labyrinthe, où l'on franchissait la hauteur des terres, on descendait le courant. Aucun incident d'importance ne marqua cette étape ${ }^{81}$.

Décidément les prédilections du missionnaire allaient "au poste chéri de Témiskaming" "sibis , lequel, du reste, le lui rendait bien. Tous les sauvages, de nouveau réunis, attendaient leur père, et le grand pavillon avait été hissé lorsque le missionnaire arriva le 26 juillet au matin. Des choses émouvantes se produisirent. Entre plusieurs autres:

C'était un sauvage arrivé là, depuis deux jours du Fort Coulonge, avec sa femme et trois enfants, dont un était

80. Ibid., 59.

81. Il faut noter ici ce qui nous paraît être une incohérence dans le texte de la relation. A la même page 64 il dit ces deux difficiles à concilier: "je partis avec trois personnes dans mon canot", et puis six lignes plus bas: "nous partâmes donc au nombre de onze dans notre canot, et nous nous rendîmes en quatre jours à Tómiskaming. Ces détails sont d'ailleurs de secondaire importance.

81 bis. M. de Bellefeuille fait seulement observer qu'on y mangea de la viande de siffleur ou marmotte du Canada ainsi que du porc-6pic. Il ajoute qu'il trouva cette viande "fort bonne, délicate approchante du poulet" ou encore identique a "celle du cochon de lait". Ibid., 64. Le sulpicien était-il un tantinet gourmet? On peut se poser la question vu l'insistance et la complaisance avec laquelle il revient plusieurs fois sur ces détails au cours de la "Relation d'une mission". 
dangereusement malade. Ce vieillard de près de 60 ans avait fait 70 lieues pour venir faire baptiser son enfant malade; mais comme je n'arrivais pas d'Abbitibbi, et que l'enfant paraissait dans un danger très prochain, un jeune homme, que j'avais instruit pour cela, l'endoya - [ sic ] - avant sa mort; et je ne doute pas qu'il ne l'ait fait d'une manière valide ${ }^{82}$.

D'autres faits de même nature marquèrent l'apostolat du missionnaire qui, durant ce nouvel arrêt de six journées à Témiskaming, baptisa 45 autres personnes dont 34 adultes, bénit deux mariages, et administra l'extrême-onction à une enfant de onze ans, la nuit qui précéda son départ.

Les résultats dépassaient les espérances du missionnaire. Aussi décida-t-il de partir, car il se faisait une obligation particulièrement grave de "missionner" au Fort des Allumettes ${ }^{83}$, où il n'avait pu faire qu'une courte apparition en 1836. Ce n'est pas que les sauvages attachés à ce poste y fussent très nombreux, -15 ou 16 familles, tout au plus, - mais, d'autre part, un bon nombre de familles chrétiennes appartenant à la mission du Lac y attendaient le missionnaire. Pour cause de pauvreté, ces bonnes gens n'avaient pu se rendre cette année-là à leur propre mission pour y remplir leur devoir de religion. Durant les 10 jours qu'il y séjourna, M. de Bellefeuille ne chôma point, ainsi qu'il nous en avertitis. La présence de ces familles déjà chrétiennes doubla son travail, car il fallut "les confesser tous et à plusieurs reprises, baptiser leurs enfants. J'ai eu l'occasion, poursuit le missionnaire, d'exercer le saint ministère en faveur de sauvages fidèles et infidèles, d'Irlandais, d'Écossais et de Canadiens français d'origine" ${ }^{84}$. En chiffres, le bilan de la mission se ramena à ceci: 19 baptêmes d'adultes sauvages; 8 d'enfants indiens; 9 enfants Irlandais un Écossais. Enfin il y eut 6 mariages de sauvages ou de métis.

M. de Bellefeuille signale enfin un problème d'importance: celui des mariages conclus entre chrétiens et infidèles, et dans lesquels

82. Ibid., 65-66. On sait que lors de la mission de l'année 1836, MM. Dupuy et de Bellefeuille s'était préoccupés de former des catéchistes indjens.

83. La "Relation d'une mission" situe ce fort de la façon suivante: le "Fort des Allumettes, qui se trouve sur la rive nord des Outawas vis-a-vis l'extrémité nord de l'śle du même nom, à environ 85 lieues plus haut que Montréal."

84. C. de Bellefeuille, "Relation d'une mission", RAPF, 2 (1840): 66. 
s'ajoutent encore les complications créées par un lien de parenté. Cet état de choses provient, nous dit-il, de ce que les sauvages sont peu nombreux et que, de ce fait, il devient difficile ou moralement impossible qu'ils ne se marient pas "entre proche-parents". De plus, comme plusieurs n'ont reçu que des rudiments de formation religieuse, ils ignorent les lois de l'Église. De là, conclut le missionnaire:

des mariages fréquents contractés chez eux entre une partie baptisée ou ondoyée et une partie infidèle, et souvent parente l'une de l'autre. C'est pourquoi je pense que le missionnaire, destiné à évangéliser ces pays, a besoin, plus que dans presque qu'aucune partie du monde, de tous les pouvoirs possibles par rapport au mariage; autrement il est exposé à rencontrer des cas capables de le jeter, lui et quelques fois ces pauvres gens dans une espèce de désespoir, ou du ımoins dans un embarras très fâcheux ${ }^{85}$.

Le 16 aout, après dix jours d'un apostolat fécond, M. de Bellefeuille quitta le poste des Allumettes, emportant en son âme la conviction que ces Indiens n'étaient et ne demeureraient infidèles que faute de prêtres en état de leur prêcher la vie chrétienne. M. Brown, le catholique bourgeois du poste, le conduisit jusqu'au Fort Coulonge. Les deux étapes suivantes furent La Passe et le Poste des Chat's, où le missionnaire arriva le 16 août, juste à temps pour prendre le bateau à destination d'Oka. Dès son arrivée au poste des Allumettes il avait renvoyé à Montréal toute son équipe de rameurs, et il n'y avait à l'accompagner à bord que des Indiens: son pilote malade, le pieux Wabimango; Michel, le déserteur de la colonie des Nipissings à Oka, que le missionnaire ramenait aux siens; les deux enfants du commis métis d'Abitibi, M. Paulson; enfin une mère sauvage avec sa fille. Ainsi encadré, M. de Bellefeuille excita vraisemblablement la curiosité, car il note avec humour qu'avec “cette suite on n'eut pas de peine à me reconnaître pour un missionnaire de sauvages".

85. Ibid., 69.

86. Ibid., 71.

87. Tel est d'après les chiffres du missionnaire lui-même le bilan des deux miscions de 1836 et 1837: 1) baptêmes: en 1836: 123 enfants, 19 adultes; en 1837: 90 enfants. 100 adultes; total des baptêmes: 332 dans les deux années. 2) Mariages: en 1836: 4; en 1837: 21; total: 25. 3) Premieres communions: en 1836: 28; en 1837:34; total 62. Ibid., 71 . 
Le 19 aout, il arrivait à la mission du Lac, fatigué mais heureux, et surtout, les mains chargées d'œuvres. Après trois jours de repos, il rentrait à Montréal qu'il avait quitté deux mois et demi plus tôt. Toutefois l'homme de Dieu n'avait encore accompli qu'une partie de sa tâche. Restaient encore à aborder les Indiens du Grand Lac; ce serait l'objet d'une troisième et dernière mission, où M. de Bellefeuille devait user les derniers lambeaux d'une santé depuis longtemps précaire.

\section{MISSION AU GRAND LAC VICTORIA (29 mai -8 septembre 1838)}

Encore plus tôt que les deux années précédentes, il laissait Lachine dès le $29 \mathrm{mai}$, et sa randonnée devant se prolonger jusqu'au 8 septembre, son absence ne durerait pas moins de 100 jours ${ }^{88}$. C'est qu'en effet la tâche à abattre se laissait pressentir longue et hérissée de difficultés. $M$. de Bellefeuille qui de nouveau serait le chroniqueur de l'expédition ${ }^{89}$, note avec un peu d'appréhension, semble-t-il, l'ampleur de l'entreprise et l'exiguitté des moyens à sa disposition:

Le 29 mai 1838, je partis de Montréal pour Lachine, où ayant fait réparer le canot, qui nous avait été prêté l'année précédente, ce qui prit presque toute la journée, je m'embarquai avec mes six hommes, pour qu'il fut dit que nous par-

88. C. de Bellefeuille, "Précis de la relation de la troisième Mission de Mr Bellefeuille à Témiskaming, Abbitibbi et Grand Lac", ] dorénavant cit́́ sous la rubrique "Précis de la relation de la troisième mission" \}, RAPF, 2 (1840): 87.

89. C'est là ce qu'atteste l'auteur de l'"Avant-Propos", RAPF, 2 (1840): 9: "Quant à la seconde relation - [ il s'agit de la seconde relation ócrite par M. de Bellefeuille. mais qui porte sur la troisième mission, celle de 1838 , effectúe par luimême en 1838 ] - elle a été abrégée et travajllée sur le brouillon qu'il n'a pas eu le temps de mettre au net: lequel brouillon nous conservons comme une relique précieuse." Telle est la principale source dont nous disposons pour évoquer les événements de l'année 1838. Pour ne pas égarer le lecteur, nous devons donc le prévenir que la lettre du texte que nous citons au cours de la troisième partie de ce travail est de l'éditeur, encore que bien souvent on puisse reconnaître, par comparaison avec le style de la "Relation d'une mission", le tour de phrase de $M$. de Bellefeuille lui-même. On peut aussi conclure que l'auteur ou l'éditeur a laissé tomber beaueoup de choses puisque la mission de 1838 a été plus longue et a duré plus longtemps que les deux autres et que, cependant, le récit en tient dans 15 pages du Rapport, alors que la "Relation d'une mission" (1837) en compte 56. 
tions ce jour-là. C'est sur cette fragile voiture qu'il me faudra faire en cinquante jours de marche quinze cent milles, et passer par cent trente-six rapides ${ }^{90}$.

La première étape dont fasse mention le Précis de la Relation d'une mission, est celle de Lachine-Témiskaming. Même le poste des Allumettes pour lequel le missionnaire avait pourtant certaine prédilection, ne comporta qu'un arrêt de quelques heures: tout juste le temps d'y engager comme catéchiste un sauvage, originaire du Lac des Deux-Montagnes, et qu'il fallut d'ailleurs remplacer peu après par un autre. Toutefois une consolation était réservée à $M$. de Bellefeuille: la compagnie de baie d'Hudson avait aménagé en chapelle un magasin qui pouvait avoir "vingt sept pieds de long sur dix-huit de large"91. Ce local, trop étroit sans doute pour qu'on espérât y loger les sauvages du Lac, les Irlandais, les Écossais et les Canadiens de passage, suffirait cependant à recevoir les Indiens attachés au poste. A la bonne heure! La chrétienté du Fort des Allumettes prenait forme et consistance.

Une courte apparition au poste de la Rivière du Moine; et, le 15 juin, les voyageurs atteignent Témiskaming. Ils étaient "en marche" depuis dix-sept jours. En somme ici encore les nouvelles sont bonnes. M. de Bellefeuille éprouve joie et fierté en écoutant le témoignage flatteur que l'un des bourgeoys du Fort croit pouvoir rendre touchant la piété, l'honnêteté de mœurs, la modération dans l'usage des boissons dont ont fait preuve les sauvages du poste. Chose plus touchante encore, semblable éloge est donné par un jeune protestant. Celui-ci, en effet, ayant effectué plusieurs voyages avec les indiens de Témiskaming et étant frappé de leur piété et de leurs bonnes mœurs, avait décidé d'enquêter sur cet état de choses. Il ' $m$ 'ajouta, note M. de Bellefeuille, que leur ayant fait quelques

90. C. de Bellefeuille, "Précis de la relation de la troisième mission", RAPF, 2 (1840): 73. Le missionnaire entreprenait d'ailleurs cette troisième mission dans des conditions de santé qui ne s'avéraient pas meilleures. On peut en juger par la lettre que lui adressait Mgr Lartigue peu de jours avant son départ: "Il lui donne pour sa troisième mission dans la partie supérieure du diocèse de nouvelles facultés touchant le mariage des infidèles... Il espère que sa santé lui permettra de visiter non seulement les postes de Témiskaming et du Grand Lac, mais encore ceux d'Abbitibbi et Népissingue, avec l'agrément de M le Supérieur." "Mgr Lartigue à M. de Bellefeuille au Séminaire de Montréal ", Montréal, 18 mai 1838, RAPQ (1945-46). 60.

91. C. de Bellefeuille "Précis de la Relation de la troisième mission", RAPF, 2 (1840): 73. 
questions, pour s'assurer s'ils avaient bien compris et retenu mes instructions, il fut surpris de l'exactitude de leurs réponses. Ces messieurs, conclut le missionnaire, se félicitent beaucoup de ce que les sauvages sont devenus bien modérés dans l'usage de la boisson: changement qu'ils n'attribuent pas à d'autre cause qu'au bienfait de la religion qui leur a été prêchée ${ }^{22}$.."

Tout cela ne laissait pas d'être consolant. L'apôtre reprit donc le travail. Cependant qu'il affectait deux de ses hommes à la construction, en voie depuis 1836 , de la minuscule chapelle, et qu'il envoyait les quatre autres rameurs au Fort des Allumettes pour y quérir des matériaux manquants et des provisions, il s'adonna aux exercices spirituels de la mission. Ce ne fut pas sans résultats: baptêmes de 26 adultes et de 12 enfants; instruction commencée de plusieurs catéchumènes; confession de tous les néophytes convertis durant les missions de 1836 et 1837; enfin plusieurs premières communions. La mission, toutefois, ne put durer plus de vingt jours: la disette commandait la dispersion. Les cinq derniers jours, M. de Bellefeuille les passa à suivre et à presser le travail de la construction de la chapelle qu'il parviendra à "faire lever et couvrir" à son retour du Grand Lac Victoria ${ }^{93}$. Et le 11 juillet, il prenait la route du lac Abitibi, vers la jeune chrétienté de l'année précédente et qu'il lui tardait de revoir.

Le trajet fut long de six jours et marqué de quelques événements qui, sans avoir une grande importance, n'en permettent pas moins d'apercevoir sur le vif les conditions dans lesquelles se faisait l'apostolat. Dans la journée du 13 juillet, les voyageurs étaient arrivés au dixième des Quinze portages lorsqu'un des rameurs qui, raconte le missionnaire, "voulut me porter sur ses épaules, me jeta à l'eau et me fit faire le plongeon: ce qui divertit beaucoup l'équipage à l'exception du porteur qui n'y entendait pas badinage"'94. La journée du 16 fut plus féconde en fortes émotions:

"J'eus ce jour là, note le chroniqueur, la plus grande frayeur que j'aie jamais éprouvé en canot. Dans le dernier de ce que l'on appelle les Trois portages, nous voulûmes nous

92. Ibid., 75 .

93. Ibid., 86 .

94. Ibid., 76. 
exempter la peine de faire une partie du troisième, en sautant tout en rond le rapide. Mais je fus vraiment epouvante en voyant le canot, que le courant entraînait rapidement, sur le point d'engloutir - [ sic 1 - dans une chute affreuse, que j'apercevais à quelques pas plus bas. Quelques uns des hommes se jettent à l'eau et s'accrochent comme ils peuvent aux roches et aux branches: les autres forcent de la perche et de l'aviron; mais ils se virent sur le point d'être vaincus par le courant. Un coup de main manqué, nous noyait dans un horrible precipice. Pour ma part, je fais bien résolution de ne jamais passer par cette voie périlleuse, et je conseille à mes successeurs, de ne point s'y risquer. Encore une fois, qu'ils remarquent bien que ce dangereux rapide est le troisième de ce qu'on appelle les trois portages, en allant à Abbitibbi, au delà de la hauteur des terres, puisqu'on suit le fil de l'eau' ${ }^{95}$.

Le 17, les voyageurs prenaient pied en cet autre poste de la Compagnie de la Baie d'Hudson, pour y bénéficier d'une réception excellente. Bourgeois et sauvages témoignèrent la joie qu'ils éprouvaient de revoir le missionnaire, ce qui faisait contraste avec l'accueil froid et embarrassant de l'année précédente ${ }^{96}$. Le séjour fut de courte durée comparativement: huit jours à peine, dont deux furent d'ailleurs à peu près perdus "les ayant passés en remède, remarque M. de Bellefeuille, car une révolution de bile m'avait mis hors de combat"97. En dépit de ce contretemps, celui-ci fit les exercices de la mission, ce qui lui valut la joie de baptiser 13 adultes et 17 enfants. Des résultats encore meilleurs auraient été sans doute obtenus, n'eut été l'exiguîté du local où devaient se donner les instructions. Plusieurs sauvages s'accommodant mal de cette insuffisance, s'en retournaient dans leurs cabanes sans profiter du passage du prêtre: "Il serait peut-être à propos, formule celui-ci, de profiter de l'occasion présente où la compagnie se dispose à bâtir une nouvelle maison pour la demeure du bourgeoys. On pourrait peut-être obtenir

95. Ibid., 77. On sait que M. de Bellefeuille ne devait pas profiter lui-même de son expérience, puisque la mort l'emporta un peu plus d'un mois après son retour en octobre 1838.

96. Ibid., 78.

97. Ibid., 81. A plusieurs reprises, il est question dans le "Précis de la Relation de la troisième mission" de fatigue, d'épuisement, de 'révolution de bile'. Ce qui permet de comprendre que les souhaits et les appréhensions de Mgr Lartigue au moment du départ de $M$. de Bellefeuille pour sa troisième mission, avaient bien quelque raison d'être. 
du gouverneur de l'Honorable Compagnie, à son retour en Canada, le printemps prochain, l'usage de la maison que l'on veut remplacer par une neuve"98. Abitibi devenait donc le troisième poste pour lequel M. de Bellefeuille convoitait d'obtenir un petit temple ${ }^{99}$, et par là même des cadres matériellement plus stables de vie chrétienne pour ses n ophytes de l'Outawais.

Deux raisons amenèrent le missionnaire à partir dès le 25 juillet: tout d'abord un état de fatigue extrême dont il espérait se remettre dans le voyage, et puis le désir d'arriver au Grand Lac ${ }^{100}$ au moment où seraient encore réunis pour la traite, les Indiens attachés à ce poste. Il partit donc avec quatre hommes de rames ${ }^{101}$, le catéchiste originaire de la mission du Lac, enfin un nouveau guide, - avec sa femme, - dont la présence était rendue nécessaire par la difficulté et l'inconnu de l'itinéraire à suivre. Neuf jours de navigation souvent ardue amenèrent $M$. de Bellefeuille au poste du Grand Lac, dont il voulait faire une nouvelle chrétienté. Les indications topographiques touchant l'itinéraire suivi deviennent vagues:

Nous fîmes ce voyage en gagnant dans l'Est par des détours sans fin, et il nous semblait la plus part - [ sic ] du temps ne faire une demi-lieue en avant que pour retourner ensuite deux lieues en arrière tellement il nous est arrive une fois qu'après avoir voyagé toute une journée nous nous trouvâmes le soir au rapport de notre guide, à deux lieues de l'endroit que nous avions quitté le matin; ensorte qu'un homme à pied aurait pu y retourner en peu d'heures. Il faut traverser un grand nombre de beaux lacs qui communiquent ensemble par une infinité de rivières grandes et petites. L'on passe par vingtcinq portages ou rapides ${ }^{102}$.

En somme tout ce qu'identifie le Précis de la relation d'une mission se ramène à quelques maigres détails: le Portage de l'ours, signalé comme dangereux et d'accès difficile; les deux rapides de la Petite

98. Ibid., 81-82.

99. Le poste des Allumettes avait déja le sien et celui de Témiskaming en powádait équivalemment un, lui aussi. (1840): 82 .

100. C. de Bellefeuille, "Précis de la Relation de la troisième mission", RAPF, 2

101. On sait qu'i en avait laissé deux à Témiskaming, qui devaient hater la construction de la chapelle.

102. C. de Bellefeuille, "Précis de la relation de la troisième mission", RAPF, 2 (1840): 82-83. 
et de la Grande Dalle, cette dernière donnée comme "une de ces magnifiques horreurs de la nature, qui font frémir en même temps qu'elles ravissent d'admiration"103; enfin une charmante petite rivière couverte d'aunages, et non autrement caractérisée. Le ler aout, veille du jour où l'on devait arriver à destination, un accident survint qui ajouta encore à la difficulté et aux mérites des voyageurs. Après avoir passé le 24e portage et alors qu'ils étaient encore à une bonne demi-journée du Grand Lac, ils crevèrent leur canot en heurtant une roche pointue. D'un seul coup sept varangues cassées et l'eau pénétrant "à gros bouillons", 104 tel fut le bilan de l'événement. On procéda à une réparation grossière des dégâts, ce qui permit à la frêle embarcation d'arriver tant bien que mal au poste, le 2 aoat, vers deux heures de l'après-midi.

Une quarantaine de sauvages déjà réunis, auxquels vinrent s'ajouter quelque dix autres, constitua l'auditoire habituel de M. de Bellefeuille pendant les six jours que dura la mission. Les excellentes dispositions de ces Indiens ainsi que de leur chef, leur assiduité et leur attention, tout cela fit que sept adultes et quatorze enfants purent être baptisés. De plus, comme à Témiskaming et au Lac Abitibi, une croix fut plantée, qui devenait comme une prise de possession des lieux par le Dieu des Chrétiens. Une ombre au tableau: "J'ai été obligé, raconte le chroniqueur, de m'élever avec force contre deux vices capitaux, l'ivrognerie et la paresse, qui dominent là plus qu'ailleurs, et sont la cause nécessaire de leur extrême pauvretét ${ }^{105}$."

Le huit aout, on prenait la route du retour. Le missionnaire ne laissait pas d'être heureux des résultats obtenus, mais il était tenaillé par une crise du foie qui l'avait amené à travailler misérablement dans les derniers jours de la mission. Un arrêt de cinq jours à Témiskaming permit de faire "lever et couvrir la chapelle" 106 commencée depuis deux ans déjà. Le nombre des chrétiens fut aussi renforcé, car quatorze autres Indiens, adultes, reçurent le baptême.

103. Ibid., 83.

104. Ibid., 84.

105. Ibid., 85.

106. Ibid., 86. 
Sans plus tarder, M. de Bellefeuille partit et arriva en quatre jours au Fort des Allurnettes. Du 24 ao át au 3 septembre, il travailla d'arrache-pied à christianiser ce poste devenu le confluent des éléments humains les plus disparates: sauvages de la mission du Lac, Indiens fidèles et infidèles, Canadiens, Écossais et Irlandais ainsi que des protestants désireux d'embrasser le catholicisme. Il peina tant et si bien que lorsqu'il quitta ces lieux, le 3 septembre, il avait "réhabilité plusieurs mariages" et baptisé au moins 39 personnes. Et ce fut ainsi que, le cœur en liesse et dans un état de dangereux affaiblissement, il arriva a Montréal, le 8 septembre, après cent jours d'absence, pour célébrer la fête du Saint Nom de Marie ${ }^{107}$.

Faisons le point. Au cours des étés de 1836, 1837 et 1838, M. de Bellefeuille avait rétabli les contacts avec des terres qui, pour avoir connu le missionnaire sous le régime français, n'en étaient pas moins redevenues infidèles. Voyageant en canot, il avait parcouru la distance approximative de 3,500 milles sur l'Outawais, et visité au moins dix postes de diverse importance et situés sur les deux rives nord et sud. C'étaient Fitz-Roy, La Passe, Fort Coulonge, l'Ile des Allumettes, les Portages de la Montagne et du Grand Marais, le rapide de la Roche-Capitaine, Témiskaming, Abitibi et le Grand Lac Victoria. De ces dix terrains d'apostolat, ces trois derniers et le poste de l'Ile des Allumettes étaient en voie d'organisation stable. Le missionnaire y avait prêché l'évangile à diverses tribus de langue algonquine

107. C'est cet affaiblissement même qui, aux dires des contemporains, a causé la mort du Missionnaire. Ainsi, par exemple, Mgr Bourget, l'auteur de l'"Avantpropos" du RAPF, 2 (1840): "Quant à Mr Charles Louis le Fèvre de Bellefeuille qui a été le digne instrument dont s'est servi la Providence pour seconder les efforts du zélé prélat - [ Mgr Lartigue, sans aucun doute ] - l'on sait qu'épuisé de fatigues, au retour de $\mathbf{8 a}$ troisième mission à Témiskaming et autres postes, il succomba à une attaque de fièvre inflammatoire a laquelle il ne put résister à cause de l'extrême faiblesse où l'avaient réduit les travaux de ses trois courses apostoliques. L'on n'aura pas de peine à s'en convaincre en lisant les deux relations qu'il nous a laissées de ses deux dernières missions, surtout si l'on fait attention que ce fervent missionnaire n'avait qu'une très faible santé et un tempérament délicat à opposer à un genre de vie si pénible." p. 9. De fait $M$. de Bellefeuille est mort le 25 octobre et inhumé le surlendemain "sous les voutes de l'Eglise paroissiale" (celles de Notre-Dame). Registres des sépultures (1838): 112e feuillet, verso, aux Archives de la Fabrique de NotreDame de Montréal. 
ainsi qu'aux blancs vivant dans ces régions. Enfin il avait administré le baptême à 550 personnes environ, et administré les sacrements de pénitence, d'eucharistie et de mariage. Bref il avait opéré tout cela avec une santé chancelante que l'entreprise n'avait laissé de ruiner complètement.

En ce faisant il avait pris, à l'instigation de Monseigneur Lartigue, une initiative apostolique qui doit être portée au crédit du très jeune diocèse de Montréal et dont les Oblats de Marie-Immaculée, établis à Bytown en l'année 1844, allaient recueillir et multiplier les fruits.

Yvon Charron, p.s.s.

\section{GOURS DE L'INSTITUT D'HISTOIRE À L'UNIVERSITÉ DE MONTREAL}

Nos lecteurs sont priés de se reporter à notre Chronique de l'Institut. Ils y verront l'annonce des cours de notre Institut pour cette année 1951. Professeur: M. Guy Frégault. Sujet général des cours: Pierre de Rigaud de Vaudreuil Cavagnal en Louisiane.

Sujets de chacun de ces cours:

16 octobre: La Louisiane

17 octobre: Politique indigène

23 octobre: La révolte des Chactas

24 octobre: Histoire administrative

Nous comptons sur la fidèle assiduité de nos lecteurs à ces cours de notre Institut. 